\title{
Preprocessing of a Fingerprint Image Captured with a Mobile Camera
}

\author{
Chulhan Lee ${ }^{1}$, Sanghoon Lee ${ }^{1}$, Jaihie Kim ${ }^{1}$, and Sung-Jae Kim² \\ 1 Biometrics Engineering Research Center, \\ Department of Electrical and Electronic Engineering, \\ Yonsei University, Seoul, Korea \\ devices@yonsei.ac.kr \\ 2 Multimedia Lab., SOC R\&D center, \\ Samsung Electronics Co., Ltd, Gyeonggi-Do, Korea
}

\begin{abstract}
A preprocessing algorithm of a fingerprint image captured with a mobile camera is proposed. Fingerprint images from a mobile camera are different from images from conventional or touch-based sensors such as optical, capacitive, and thermal sensors. For example, images from a mobile camera are colored and the backgrounds or non-finger regions can be very erratic depending on how the image captures time and place. Also, the contrast between the ridges and valleys of images from a mobile camera is lower than that of images from touch-based sensors. Because of these differences between the input images, a new and modified fingerprint preprocessing algorithm is required for fingerprint recognition when using images captured with a mobile camera.
\end{abstract}

\section{Introduction}

Mobile products are used in various applications such as communication devices, digital cameras, schedule management devices, and mobile banking. Due to the proliferation of these products, privacy protection is becoming more important. Fingerprint recognition has been the most widely exploited because of stability, usability, and low cost. There are already a few commercial mobile products equipped with fingerprint recognition systems. However, these products require additional fingerprint sensors. This leads to weakening durability and increasing price. Fortunately, almost all modern mobile products have high computational power and are already equipped with color cameras. These cameras are comparable in quality to commercial digital cameras, with features such as zooming, auto-focusing, and high resolution. Because of hardware contributions(high computational power and camera) and privacy protection problems in mobile environments, a new fingerprint recognition system which uses these kinds of mobile camera devices is realizable in near future. There are many challenges when developing fingerprint recognition systems which use a mobile camera. First, the contrast between the ridges and the valleys in the images is lower than that in images obtained with touch-based sensors. Second, because the depth of field of 
the camera is small, some parts of the fingerprint regions are in focus but some parts are out of focus. Third, the backgrounds, or non-finger regions, in mobile camera images are very erratic depending on how the image captures place and time. For these reasons, a new and modified preprocessing algorithm is required. In Section 2, we explain how we obtained the fingerprint image for our work and explain the segmentation algorithm. Section 3 presents the orientation estimation. Experimental results are shown in Section 4, followed by a conclusion and future work in Section 5 .

\section{Fingerprint Segmentation}

Firstly, we explain how we obtained the fingerprint images for our work. We used an acquisition device composed of a 1.3M pixel CMOS camera used on a mobile phone and a LED (Light Emitting Diode). The working distance was set as $5 \mathrm{~cm}$ in front of the camera, and a finger was positioned here to get fingerprint images with a additional holder. Because of the LED, we were able to obtain fingerprint images which are less affected by outside light conditions. After acquiring a fingerprint image with a mobile camera, the first step is fingerprint segmentation. This process divides the input image into a foreground (fingerprint) region and a background region. When a fingerprint image is obtained from a touch-based sensor such as a capacity, optical, or thermal sensor, the background or nonfinger region is easy to segment from the fingerprint region because the region has similar patterns depending on sensor types. However, when a fingerprint is captured by a mobile camera, the background regions are very erratic depending on how the image captures place and time.

\subsection{Fingerprint Segmentation Using Color Information}

In order to segment fingerprint regions using color information, we compare each pixel in the input image with the distribution of the fingerprint color model in the normalized color space. [1] shows that even though the skin color of humans is different from that of the entire human species according to each melanin, the skin color of the palm (including the fingers) is mainly influenced by an absorption spectrum of oxygenated hemoglobin because of the absence of melanin at the palm. Therefore, the fingers of all humans show similar reflection rates according to visual wavelengths. With this characteristic, the normalized color distribution which we determined with our sample images can be applied to all humans. In this paper, we model fingerprint color distribution with a nonparametric modeling method using a lookup table (LUT) 2]. We produced 100 training images and 400 test images by manually segmentation. One of the training images is shown in Fig. 1(a),(b). With the training images, the foreground regions are transferred to normalized rgb color space and then accumulate the normalized $\mathrm{r}, \mathrm{b}$ information in order to make the distribution of fingerprint color(Fig 1(c)). To create the LUT, the color space (rb space) is quantized into a number of cells according to a predefined resolution and the value of each 


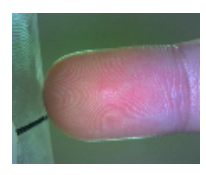

(a)

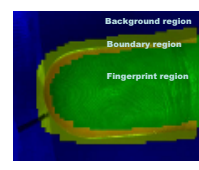

(b)

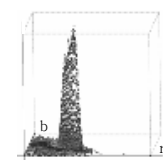

(c)

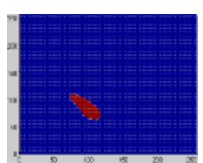

(d)

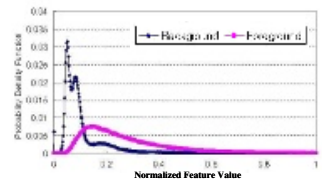

(e)

Fig. 1. (a) Original Image, (b) Manually Segmented Image (c)The distribution of the fingerprint color model, (d) The LUT with $256 \times 256$ Resolution (e) The distribution of Tenengrad-based measurement

cell is divided by the largest value. We categorize the cells as fingerprint region cells if the divided value is larger than $T_{L U T}$. If not, the cells represent background region cells. We experimentally define $T_{L U T}$ as 0.1 . Fig. $1(\mathrm{~d})$ shows the LUT with $256 \times 256$ resolution. With the LUT, each pixel $x(i, j)$ is segmented as follows:

$$
x(i, j)=\left\{\begin{array}{c}
\text { fingerprint_region_if_LUT }[r(i, j)][b(i, j)]=\text { fingerprint_cell } \\
\text { background_region_if_LUT }[r(i, j)][b(i, j)]=\text { background_cell }
\end{array}\right.
$$

where $\mathrm{r}(\mathrm{i}, \mathrm{j})$ and $\mathrm{b}(\mathrm{i}, \mathrm{j})$ are the normalized $\mathrm{r}$ and $\mathrm{b}$ values of a pixel $x(i, j)$. To reduce noise, we apply this process to each block. Each block is represented by the average $r$ and $b$ values within the blocks of predefined size $(8 \times 8)$.

\subsection{Fingerprint Segmentation Using Frequency Information}

In order to capture a fingerprint image with a camera, a close-up shot is required. This makes the depth of field (Dof) small. This means that the fingerprint region is in focus and the background region is out of focus, which produces clear ridge-pattern images in the fingerprint region and blurred-pattern images in the background region. Our method is based on this difference between the two image regions. We consider the Tenegrad based method that has been exploited in the auto-focusing technique 3. In the Tenengrad based method, using the Sobel operator, we calculate the horizontal $\left(G_{H}\right)$ and $\operatorname{vertical}\left(G_{V}\right)$ gradients of the images. Our Tenengrad based measurements are determined as follows:

$$
\operatorname{Tenengrad}(i, j)=\frac{1}{(2 n+1)^{2}} \sum_{k=i-n}^{i+n} \sum_{l=i-n}^{i+n} G_{V}^{2}(k, l)+G_{H}^{2}(k, l)
$$

Fig. 1(e) show the distributions of the measurement of the fingerprint region and the background region with manually segmented images (the training images in Section 3.1). The distribution shows that the measured values of the background region are concentrated on low values and the values of the fingerprint region 
are spread out wildly. Taking advantage of these characteristics, segmentation is achieved through the simple threshold method. The threshold is determined by Bayesian theory with two distributions, the background distribution and the foreground distribution. In Bayesian theory, we assume that a priori probabilities are the same.

\subsection{Fingerprint Segmentation Using the Region Growing}

The final fingerprint segmentation algorithm is conducted with the region growing method. In the region growing algorithm [4, the seed region and the similarity measurement (which merges neighboring pixels) must be determined. To determine the seed region, we combine the results of color (Section 2.1) and frequency (Section 2.2) with the AND operator. This is because the fingerprint region should be well focused, and also because it should show the finger color. From the determined seed region, we estimate the color distribution of each input finger as the color distribution of the seed region. With the color distribution, the similarity measurement is defined as follows:

$$
\begin{aligned}
& \mathbf{D}(i, j)=(\mathbf{x}(i, j)-\mathbf{m})^{\mathbf{T}} \Sigma^{-1}(\mathbf{x}(i, j)-\mathbf{m}) \\
& (i, j) \text { is_fingerprint_region : if_D }(i, j)<\mathrm{T}_{\mathbf{S}} \\
& (i, j) \text { is_background_region : otherwise }
\end{aligned}
$$

, where $\mathbf{x}(\mathbf{i}, \mathbf{j})$ is the normalized $\mathrm{r}$ and $\mathrm{b}$ value of a neighbor pixel that will be merged. $\mathbf{m}$ and $\Sigma$ are the means of the normalized $\mathrm{r}$ and $\mathrm{b}$ values and the covariance matrix calculated within the seed region. Fig. 2 shows the resulting images of color, frequency, combining color and frequency, and final segmentation $\left(T_{s}=4\right)$. In Section 5, the proposed segmentation algorithm is evaluated by manually segmented images.

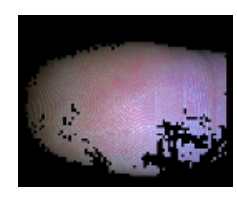

(a)

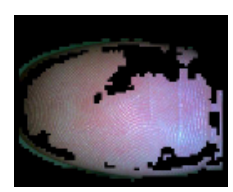

(b)

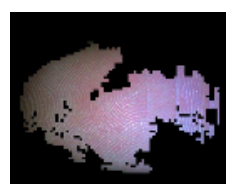

(c)

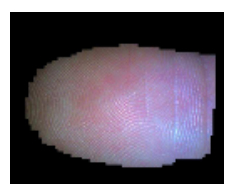

(d)

Fig. 2. The resulting images: (a) Color (b) frequency (c) Combining (d) Final segmentation $\left(T_{s}=4\right)$

\section{Fingerprint Orientation Estimation}

Many algorithms have been proposed for orientation estimation. Among these, gradient-based approaches [5] 6] are the most popular because of low computational complexity. However, gradient-based approaches are very sensitive to noise, especially non-white Gaussian noise in the gradient field because it is based on least square method. In this section, we propose the robust orientation estimation method based on iterative regression method. 


\subsection{Orientation Estimation Based on the Iterative Robust Regression Method}

In fingerprint images captured with a mobile camera, since the contrast between ridges and valleys is low, outliers are caused by not only scars on specific fingerprints but also by camera noise. To overcome the problem of outliers, we apply the robust regression method. This method tends to ignore the residuals associated with the outliers and produce essentially the same results as the conventional gradient based method when the underlying distribution is normal and there are no outliers. The main steps of the algorithm include:

i) 2-Dimensional gradients $\left(\mathbf{x}_{i}=\left[G_{x}, G_{y}\right]\right)$ : An input image is divided into subblocks, and the 2-Dimensional gradients are calculated using the Sobel operator.

ii) Orientation estimation: Using the calculated 2-Dimensional gradients, the orientation of the sub-block is estimated by the conventional gradient method.

iii) Whitening: The gradients $\left(\mathbf{x}_{i}\right)$ are whitened to measure a norm in the Euclidean space.

iv) Removing outliers: In the whitened 2-Dimensional gradients field, a gradient $\mathbf{x}_{i}$ is removed if the Euclidean norm of the whitened gradient $\left(\left\|\mathbf{x}_{i}\right\|_{w}\right)$ is larger than $2 \sigma$, where $\sigma$ is 1 because of whitening.

v) Orientation re-estimation: Using the 2-Dimensional gradients from step 4, the orientation $(\theta(n+1))$ of the sub-block is re-estimated by the conventional gradient method.

vi) Iterative procedure: If $|\theta(n+1)-\theta(n)|$ is less than $T_{\theta}$, the procedure is stopped. If not, we revert to step 3 . The $T_{\theta}$ is defined according to quantized Gabor filter orientation that is used on ridge enhancement.

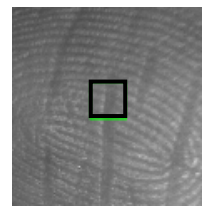

(a)

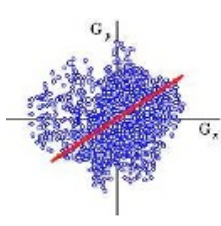

(b)

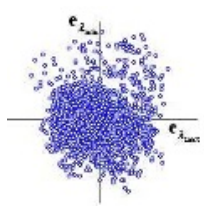

(c)

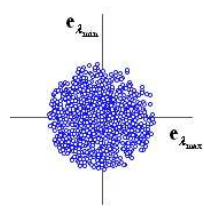

(d)

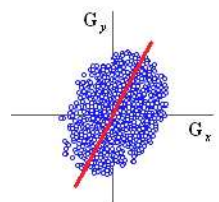

(e)

Fig. 3. (a) A sub-block image (b) A 2D gradient field with outliers (c) A whitened 2D gradient field (d) whitened $2 \mathrm{D}$ gradient field without outliers (e) A $2 \mathrm{D}$ gradient field without outliers

Since the gradient elements corresponding to the outliers have an influence on the orientation estimation, they have relatively larger Euclidean norm values than those corresponding to the ridges in the whitened gradient field. So, the gradient elements corresponding to the outliers are removed by comparing the norms of the gradient elements on step iv). Fig. 3 shows the result of our proposed algorithm schematically. The ridge orientation in the sub-block is represented by 
the orthogonal direction to the line shown in (b) and (e). The line in (b) is pulled by the outliers caused by the scar. After removing the outliers in the gradient field, the line in (e) represents the reliable direction.

\section{Experimental Result}

\subsection{Segmentation}

400 test images from 150 different fingers were evaluated in terms of segmentation. Each test image was manually separated into fingerprint regions and background regions. To evaluate the segmentation algorithm, we compared the output of the proposed segmentation method with the manually labeled results. We created 4 different resolution LUTs $(256 \times 256,128 \times 128,64 \times 64,32 \times 32)$ and calculated the error according to merging-threshold $T_{s}$. There are two types of error: a type I error which misjudges the fingerprint region as the background region, or a type II error which misjudges the background region as the fingerprint region. Fig. 4(a) shows the total error (type I + type II) curve. Here, the horizontal axis represents the value of merging-threshold $T_{s}$, and the vertical axis is the error rate. Fig. 4(a) indicates that we get the best segmentation performance when $T_{s}$ is between 4 and 5 , and better segmentation performance when larger resolution LUTs are used. When $T_{s}$ is less than 4 , the type I error increases and the type II error decreases. When $T_{s}$ is greater than 5 , the type I error decreases and the type II error increases.

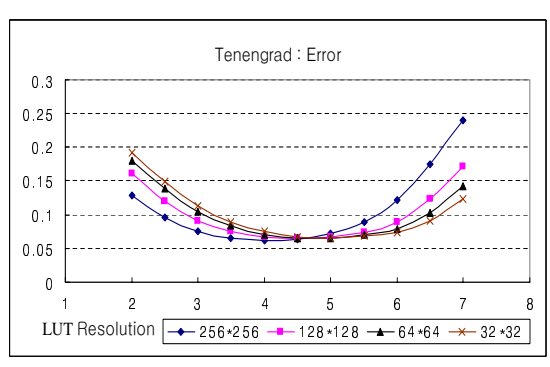

(a)

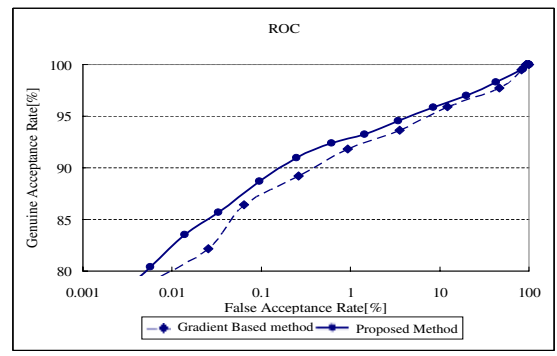

(b)

Fig. 4. (a)Fingerprint segmentation total error curve (b)The ROC curve of the gradient-based method and the proposed method

\subsection{Orientation Estimation}

We compared the orientation estimation methods with verification performance. To evaluate verification performance, we applied the proposed segmentation algorithm and implemented a minutia extraction [7] and a matching algorithm [8]. In this experiment, we used a fingerprint database of 840 fingerprint images 
from 168 different fingers with 5 fingerprint images for each finger. We compared the verification performance after applying a conventional gradient-based method and the proposed method for orientation estimation. Fig. 4(b) shows the matching results with the ROC curve. We can observe that the performance of the fingerprint verification system is improved when the proposed orientation method is applied.

\section{Conclusion and Future Work}

In this paper, we propose a fingerprint preprocessing algorithm using a fingerprint image captured with a mobile camera. Since the characteristics of fingerprint images acquired with mobile cameras are quite different from those obtained by conventional touch-based sensors, it is necessary to develop new and modified fingerprint preprocessing algorithms. The main contributions of this paper are the method of fingerprint segmentation and the robust orientation estimation algorithm when using a mobile camera. In future work, we will develop the matching algorithm that is invariant to 3D camera viewpoint change in mobile camera images and compare fingerprint recognition system in images captured with mobile cameras and touch-based sensors. In this comparison, we will compare not only verification performance but also image quality, the convenience of usage and the number of true minutiae.

\section{Acknowledgements}

This work was supported by Samsung Electronics Co. Ltd. and Korea Science and Engineering Foundation (KOSEF) through the Biometrics Engineering Research Center at Yonsei University.

\section{References}

1. Angelopoulou Elli, "Understanding the Color of Human Skin. "Proceedings of the 2001 SPIE conference on Human Vision and Electronic Imaging VI, SPIE Vol. 4299, pp. 243-251, May 2001.

2. Zarit, B. D., Super, B. J., and Quek, F.K.H. "Comparison of Five Color Models in Skin Pixel Classification" International Workshop on Recognition, Analysis, and Tracking of Faces and Gestures in Real-Time Systems, pp. 58-63, 1999.

3. NK Chern, PA Neow, MH Ang Jr. "Practical issues in pixel-based autofocusing for machine vision", Int. Conf. On Robotics and Automation, pp. 2791- 2796, 2001.

4. R. C. Gonzalez, R. E. Woods, "Digital Image Processing", Addison-Wesley, Second Edition, pp. 613, 2002.

5. Nalini K., Ratha, Chen Shaoyun, Anil K. Jain, "Adaptive flow orientation-based feature extraction in fingerprint images", Pattern Recognition, Vol. 28, Issue 11, pp. 1657-1672, November 1995. 
6. A.M. Bazen and S.H. Gerez, "Directional field computation for fingerprints based on the principal component analysis of local gradients", in Proceedings of ProRISC2000, 11th Annual Workshop on Circuits, Systems and Signal Processing, Veldhoven, The Netherlands, November 2000.

7. L. Hong, Y. Wan and A.K. Jain, "Fingerprint Image Enhancement: Algorithms and Performance Evaluation", IEEE Transactions on PAMI, Vol. 20, No. 8, pp.777-789, August 1998.

8. D. Lee, K. Choi and Jaihie Kim, "A Robust Fingerprint Matching Algorithm Using Local Alignment", International Conference on Pattern Recognition, Quebec, Canada, August 2002. 